\title{
Optimized transmission-line impedance transformers for petawatt-class pulsed-power accelerators
}

\author{
D. R. Welch, T. C. Genoni, D. V. Rose, and N. L. Bruner \\ Voss Scientific, LLC, Albuquerque, New Mexico 87108, USA
}

\author{
W. A. Stygar
}

Sandia National Laboratories, Albuquerque, New Mexico 87185, USA

(Received 7 December 2007; published 17 March 2008)

\begin{abstract}
We have developed 1D analytic and 2D fully electromagnetic models of radial transmission-line impedance transformers. The models have been used to quantify the power-transport efficiency and pulse sharpening of such transformers as a function of voltage pulse width and impedance profile. For the cases considered, we find that in the limit as $\Gamma \rightarrow 0$ (where $\Gamma$ is the ratio of the pulse width to the one-way transit time of the transformer), the transport efficiency is maximized when the impedance profile is exponential. As $\Gamma$ increases from zero, the optimum profile gradually deviates from an exponential. A numerical procedure is presented that determines the optimum profile for a given pulse shape and width. The procedure can be applied to optimize the design of impedance transformers used in petawatt-class pulsedpower accelerators.
\end{abstract}

DOI: 10.1103/PhysRevSTAB.11.030401

PACS numbers: $84.70 .+\mathrm{p}, 84.60 . \mathrm{Ve}, 52.58 . \mathrm{Lq}$

\section{INTRODUCTION}

Radial transmission-line impedance transformers provide an efficient method of combining the outputs of as many as several hundred traditional Marx generators or linear-transformer-driver (LTD) modules to produce a petawatt-level power pulse [1]. Modular pulsed-power systems making use of LTD technology [2,3] have been proposed for versatile transmission line and a wide range of applications including z-pinch experiments [4], flash radiography [5], and excimer lasers [6] for inertial fusion energy. An LTD module is a compact inductive voltage adder in which each of the adder's cavities is driven by capacitors and switches that are located within the cavity. This technology may offer an attractive alternative to traditional Marx-generator systems, such as that currently being used in the 55-TW Z accelerator [7] at Sandia National Laboratories.

In a recently published design of a petawatt-class $z$-pinch accelerator [1], LTD modules are used to drive monolithic water-insulated radial transmission lines with an exponentially increasing impedance. The design is outlined in Fig. 1. Through the interface between the LTDs and the water-filled transformers, bunches of 7 LTD modules drive 2 of the 60 total transformers which consist of parallel plates broken in azimuth as shown. In the complex 3D convolution, the LTD electrodes connect to two levels of transformers at a given azimuth. At their outputs, the impedance transformers are connected to magnetically insulated transmission lines (MITLs). These are combined in parallel at small radius by a vacuum posthole convolute. Finally, the output of the convolute is a short single MITL that delivers current to the $z$-pinch load. The transformers also serve as passive high-pass filters that reduce the amplitude of any low-frequency prepulse that might exist, and in addition, compress slightly the electrical power pulse. This characteristic may reduce the need for additional pulse sharpening components and make an accelerator design more compact.

In the design of Ref. [1], the transformer impedance profile is assumed to be exponential. In an exponential profile the fractional change in impedance per unit length is constant; i.e., $Z_{f}^{-1} d Z_{f} / d r=$ constant [8]. Such a profile has been found to be more efficient than, for example, a linear impedance profile $\left(d Z_{f} / d r=\right.$ constant) $[9,10]$; however, it appears that the impedance profile that optimizes power transmission has not been previously established. Since a future petawatt-class pulsed-power accelerator would likely require a substantial effort to design and build, it is of interest to maximize the accelerator's efficiency, which would include determining the im-

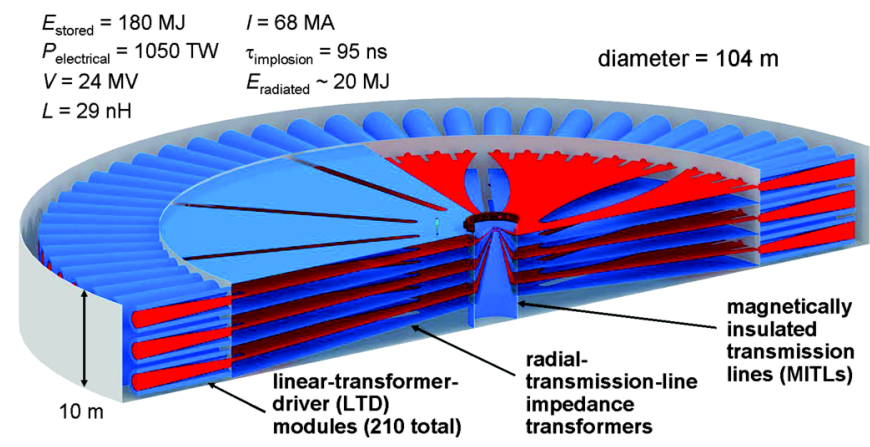

FIG. 1. (Color) Proposed architecture of a petawatt-class pulsedpower accelerator for $z$-pinch experiments in Ref. [1]. The LTD modules drive the radial-transmission-line impedance transformers, which in turn drive the MITLs, and ultimately, the $z$-pinch load in the center of the accelerator. 
pedance profiles that maximize the efficiencies of the accelerator's impedance transformers.

In this paper, we examine the behavior of idealized transmission-line impedance transformers, both analytically and in the first 2D fully electromagnetic simulations of such transformers. In Sec. II, we present an analytic 1D model of a transmission line with an arbitrary impedance profile, and calculate the power efficiency and pulse sharpening for several different profiles. Section III describes the electromagnetic simulations of the geometry and accelerator characteristics shown in Fig. 1, as well as that of the alternate profiles discussed in Sec. II. Results predicted by the analytic model and numerical simulations are presented in Sec. IV. We also present in Sec. IV a numerical technique that can be applied to determine the impedance profile that optimizes a transformer's power-transport efficiency, for a given pulse shape and width. A summary of the work is presented in Sec. V. An alternate method for calculating the transformer power efficiency using a series of transmission-line elements is presented in the Appendix.

\section{ANALYTIC MODEL OF A RADIAL- TRANSMISSION-LINE IMPEDANCE TRANSFORMER}

In this section, we describe the transformer characterization and analytic technique used to model the pulse transmission properties of an idealized 1D radial-line impedance transformer.

A schematic of the geometry under consideration is shown in Fig. 2. The input impedance is $Z_{\text {in }}$ (at radius $r_{2}$ ); the output impedance is $Z_{\text {out }}$ (at radius $r_{1}$ ). The transformer gap $h(r)$ is in general a function of radius. We assume that the transformer is impedance matched to a constant-impedance line at each end.

In the quasi-TEM mode approximation [11], the propagating voltage wave in the frequency domain is governed by the model equation,

$$
\frac{1}{r} \frac{d}{d r}\left(r \frac{d V}{d r}\right)-\frac{1}{r Z} \frac{d(r Z)}{d r} \frac{d V}{d r}+k^{2} V=0,
$$

where $k=\omega / c$ is the wave number,

$$
Z=Z_{0} \frac{h(r)}{2 \pi r},
$$

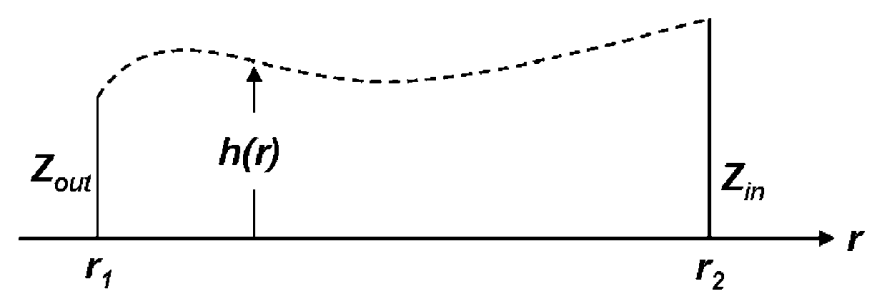

FIG. 2. Schematic of the radial-transmission-line impedancetransformer geometry used by the analytic model. The function $h(r)$ is the transmission-line gap as a function of $r$. and the impedance $Z_{0}=\sqrt{\mu / \varepsilon}$, where the permeability $\mu$ and the permittivity $\varepsilon$ are those of the transformer medium. When the transformer gap $h(r)$ is a constant, Eq. (1) reduces to Bessel's equation and the propagating solutions are Hankel functions of the first and second kind. A complete discussion of the modes and transmission and reflection properties of a radial transmission line with a constant gap can be found in Ref. [12].

Expanding the derivative of $r Z$ in Eq. (1), we see that the governing equation for the voltage reduces to

$$
\frac{d^{2} V}{d x^{2}}-\frac{1}{Z} \frac{d(Z)}{d x} \frac{d V}{d x}+k^{2} V=0
$$

which is identical in form to the model equation for the voltage in a planar transmission line, as it must be, and $r$ and $x$ are interchangeable when operating in cylindrical or Cartesian coordinate systems. Hence, we can carry out our analysis in the simpler Cartesian geometry keeping in mind the additional factor of $1 / r$ in the impedance of the radial line. For a constant-impedance line, solutions for $V$ are plane waves $e^{i k x}$ (or cylindrical waves $e^{i k r}$ in radial geometry) which produce no reflection in the quasi-TEM approximation.

For short pulses and a variable impedance line, an exponential impedance profile, which corresponds to

$$
\frac{1}{Z} \frac{d Z}{d x}=\text { constant, }
$$

has been shown to be more efficient for power transmission than the linear impedance profile $[9,10]\left(\frac{d Z}{d r}=\right.$ constant $)$ and several other functional forms [11], some of which are designed to reduce the discontinuities in slope of the input and output ends. The calculation of power transmission efficiency is necessarily numerical and depends on assumptions regarding the input pulse shape and width. Thus, a general analytic expression for the impedance profile that optimizes power transmission is not possible.

We find below, however, that in the limit as $\Gamma \rightarrow 0$, where $\Gamma$ is the ratio of the pulse width to the transformer's one-way transit time, the optimal impedance profile is an exponential function of distance. We also find that as $\Gamma$ increases from 0 , the optimal profile deviates gradually from an exponential. Significant deviations from an exponential profile are found for $\Gamma>0.5$.

\section{A. Semianalytic approach}

In the numerical calculations and 2D electromagnetic simulations discussed below, we assume an input voltage with peak $V_{0}$ of the form

$$
V_{\text {in }}(t)= \begin{cases}0, & t \leq 0 \\ V_{0} \sin \left(\omega_{0} t\right), & 0 \leq t \leq \frac{\pi}{\omega_{0}} \\ 0, & t \geq \frac{\pi}{\omega_{0}},\end{cases}
$$

where $\omega_{0}$ determines the full-width-at-half maximum 
(FWHM) $T$ of the pulse according to $T=2 \pi / 3 \omega_{0}$. The Fourier transform of $V_{\text {in }}(t)$ is given by

$$
\begin{aligned}
V_{\text {in }}(\omega) & =\int_{0}^{\pi / \omega_{0}} V_{0} \sin \left(\omega_{0} t\right) e^{i \omega t} d t \\
& =-V_{0} \omega_{0} \frac{e^{i \pi\left(\omega / \omega_{0}\right)}+1}{\omega^{2}-\omega_{0}^{2}} .
\end{aligned}
$$

Our approach is to calculate the voltage transfer function [via a solution of Eq. (4)] as a function of frequency. We assume that the inlet and outlet of the transformer are connected to semi-infinite uniform planar transmission lines with impedances matched to the input and output impedances of the transformer. In the inlet region, we prescribe an incoming plane wave of unit amplitude, $e^{i k x}$, and a reflected wave $V_{r} e^{-i k x}$. In the outlet region, the transmitted plane wave is taken as $V_{t} e^{i k(x-L)}$. The boundary conditions then take the form

$$
\begin{aligned}
& \frac{d V}{d x}+i k V=2 i k, \quad \text { at } x=0 \\
& \frac{d V}{d x}-i k V=0, \quad \text { at } x=L .
\end{aligned}
$$

Discretized on a uniform grid in $x$ to second order, Eq. (3) takes the usual form

$$
\begin{aligned}
& \frac{V_{i+1}-2 V_{i}+V_{i-1}}{\Delta x^{2}}-\frac{1}{Z_{i}}\left(\frac{Z_{i+1}-Z_{i-1}}{2 \Delta x}\right)\left(\frac{V_{i+1}-V_{i-1}}{2 \Delta x}\right) \\
& +k^{2} V_{i}=0 .
\end{aligned}
$$

Making use of the boundary conditions in Eq. (7) (after similar discretization), we obtain a linear system for the unknown values of the voltage Fourier transform $V_{i}$ at prescribed grid locations $x_{i}, 0 \leq x_{i} \leq L$. A minimum of 200 points per cycle of the oscillatory solution are used. The solution for $V(L)$ (at each frequency $\omega$ ) is obtained using a standard tridiagonal matrix algorithm [13]. Since we chose a unit amplitude input, this procedure results in a dimensionless transfer function $V_{t}(\omega)$, from which the output voltage is calculated by inverse Fourier transformation, i.e.,

$$
V_{\text {out }}(t)=\frac{1}{2 \pi} \int_{-\infty}^{\infty} V_{\text {in }}(\omega) V_{t}(\omega) e^{-i \omega t} d \omega
$$

In the special case of the exponential profile, the solutions to Eq. (3) are damped plane waves and the voltage transfer function is given analytically by

$$
V_{t}^{\exp }(\omega)=\frac{e^{\delta L / 2}}{\cos (\gamma L)-i k \sin (\gamma L) / \gamma},
$$

where $\delta=\log \left(Z_{\text {out }} / Z_{\text {in }}\right) L^{-1}$ and $\gamma^{2}=k^{2}-\delta^{2} / 4$, In general, however, $V_{t}(\omega)$ must be tabulated from the numerical solution of Eq. (3) as a function of $\omega$ with boundary conditions given by Eq. (7).

\section{B. Parametrization of impedance profiles}

In the numerical examples presented in Sec. IV, the following parameterization of the impedance profile is used,

$$
Z(x)=A+B e^{\alpha x},
$$

where $\alpha=m \delta$. For the radial line, we must replace $x$ with $r_{2}-r$ and keep in mind the additional factor of $1 / r$ in calculating the transmission-line gap profile $h(r)$,

$$
h(r)=h_{\text {in }} \frac{r Z(r)}{r_{2} Z_{\text {in }}},
$$

where $h_{\text {in }}$ is the gap at the inlet. When $m=1$ the profile is exponential:

$$
Z^{\exp }=Z_{\text {in }} e^{\delta x} .
$$

In the limit as $m \rightarrow 0$, we obtain the linear impedance profile,

$$
Z^{\text {lin }}=Z_{\text {in }}+\left(Z_{\text {out }}-Z_{\text {in }}\right) \frac{x}{L} .
$$

Values of $m>1$ and $m<1$ give profiles for which the largest relative changes in impedance occur near the outlet and inlet, respectively. In the numerical examples that follow, we consider values of $m$ in the range $[-2,2]$.

\section{DESCRIPTION OF 2D ELECTROMAGNETIC SIMULATIONS OF THE TRANSFORMER}

In order to test thoroughly the quasi-TEM-mode approximation of the theory, we conduct $2 \mathrm{D}$ cylindrical simulations of a radial transmission line using the fully relativistic electromagnetic code LSP [14]. We use two different electromagnetic field solution techniques: an optional implicit field-solving algorithm [15] (to relax Courant in this case by $40 \%$ ) and the standard explicit (leapfrog) electromagnetic solver. A comparison of the two field solvers using the exponential impedance profile (design I discussed in the next section) shows negligible differences. Figure 3 gives an example of the radial transmission-line geometry used in simulation for the transformer design I case (described in the next section) with $m=0$; i.e., a linear impedance profile. A forwardgoing TEM voltage wave is fed into the transmission line at large radius and propagates through the water-filled $(\varepsilon=$ 80) cavity toward the load, exiting at small radius. To ensure that the simulation region near the outlet boundaries (simple wave-transmitting boundaries) well represents the injected TEM wave structure, and to avoid rapidly changing impedances at the outlet, $50-\mathrm{cm}$ matching sections (constant gap) are placed just upstream of $r_{2}$ and downstream of $r_{1}$. The simulation impedances at $r_{2}(3688 \mathrm{~cm})$ and $r_{1}(305 \mathrm{~cm})$ are 0.203 and $2.16 \Omega$, respectively [1]. The forward-going pulse is modeled as in Eq. (5).

The LSP code uses a structured orthogonal grid that necessitates a "stair stepping" of the cathode and anode 


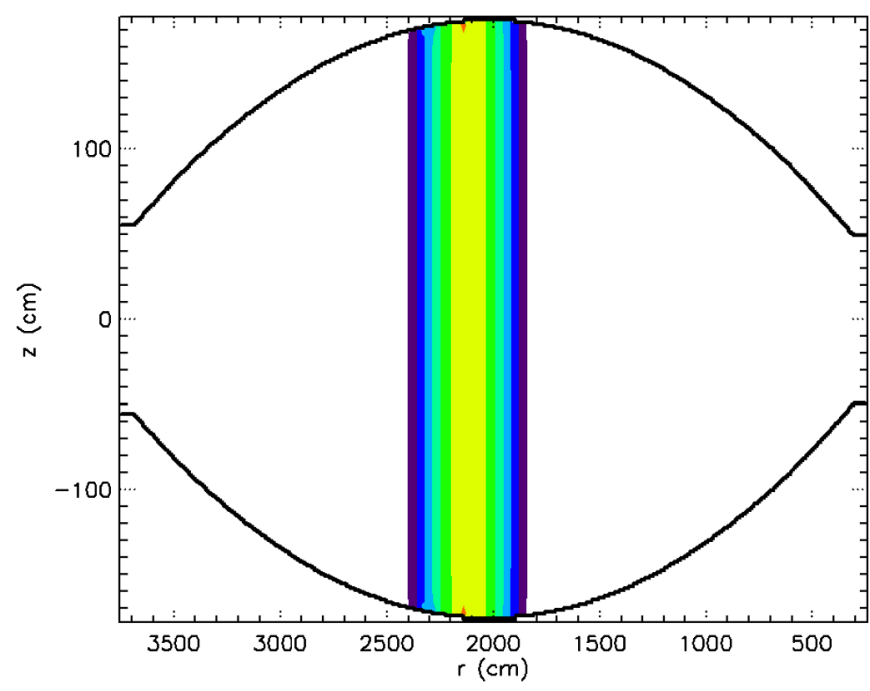

FIG. 3. (Color) Geometry of the radial-transmission-line impedance transformer used in the 2D $(r, z)$ LsP simulation for $m=0$ (linear impedance profile) showing the propagating electromagnetic pulse after $450 \mathrm{~ns}$.

surfaces as seen in Fig. 3 which could yield spurious reflections. We found that, given the $30-\mathrm{m}$ radial scale and 1-m axial scale length, cell sizes of $\Delta r=6.6 \mathrm{~cm}$ and $\Delta z=1.7 \mathrm{~cm}$ provide reasonably accurate results. Indeed, with an exponential impedance profile, the use of a 4 times larger cell size in both directions results in $<1 \%$ change in the peak of the output wave.

\section{RESULTS FROM THE SEMIANALYTIC AND 2D SIMULATIONS}

Making use of the techniques described in Secs. II and III, we investigate in this section the power-transport efficiency and other characteristics of the transformer of the two published accelerator designs.

\section{A. Transformer design I}

We investigate two water-insulated transformer designs under consideration for the next generation of petawattclass $z$-pinch drivers [1]. Parameters of the first design are $r_{2}=3688 \mathrm{~cm}, Z_{\text {in }}=0.203 \Omega, r_{1}=304.8 \mathrm{~cm}$, and $Z_{\text {out }}=2.16 \Omega$, which give a one-way transit time $\tau=$ 1009 ns. Semianalytic calculations and 2D simulations are carried out for impedance profiles corresponding to $m=2,1,0,-1$, and -2 in Eq. (10). The gap profiles are found from Eq. (11) with $h_{\text {in }}=112 \mathrm{~cm}$. The radial and equivalent planar transmission-line profiles are shown in Figs. 4 and 5 respectively.

The input voltage is of the form Eq. (5) with $\omega_{0}=1.4 \times$ $10^{7} \mathrm{~s}^{-1}$. This corresponds to a ratio $\Gamma \equiv T / \tau \simeq 0.15$, comfortably in the high-frequency regime. (We consider the effect of longer $T$ in the second design.) Numerical solutions of Eqs. (3) and (7)-(9) are obtained for the same design parameters and the equivalent gap profiles of Fig. 4.

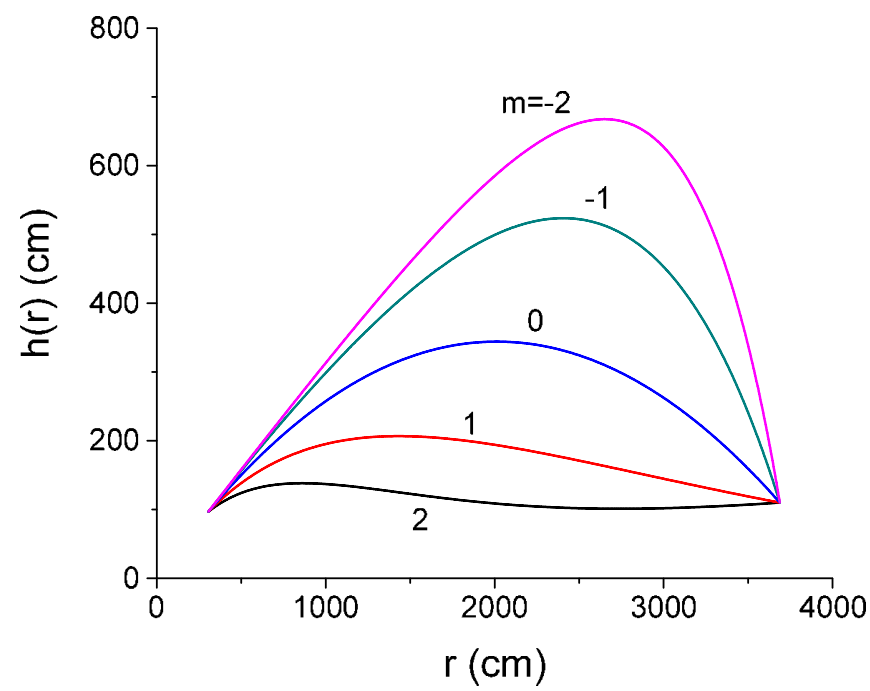

FIG. 4. (Color) Gap profiles $h(r)$ as a function of $r$ for transformer design I and several values of the gap parameter $m$.

From the results presented in Table I, we see that the 1D numerical results based on the quasi-TEM approximation agree to $1 \%$ with the $2 \mathrm{D}$ electromagnetic simulations. Each show that the exponential profile $(m=1)$ gives the highest power transmission efficiency (confirming the value chosen in Ref. [1]).

\section{B. Transformer design II}

An alternative transformer design from Ref. [1] was chosen for a series of numerical calculations to investigate the effect of pulse length (as measured by the ratio $\Gamma$ ) and shape, in addition to a more systematic study of the optimization of the impedance profile. The parameters for

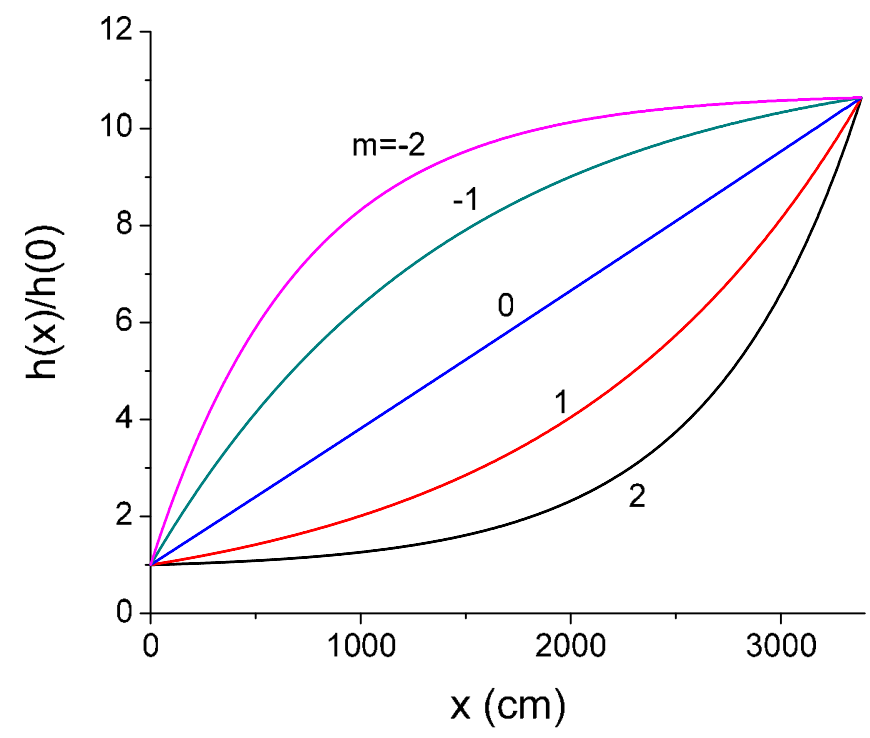

FIG. 5. (Color) Equivalent planar-transmission-line gap profiles of the normalized gap $h(x) / h(0)$ as a function of $x$. 
TABLE I. Power transmission efficiencies of the design I impedance transformer with various impedance profiles from Eq. (10).

\begin{tabular}{ccc}
\hline \hline $\begin{array}{c}\text { Profile } \\
\text { parameter }(m)\end{array}$ & $\begin{array}{c}\text { ID numerical } \\
\text { efficiency }(\%)\end{array}$ & $\begin{array}{r}\text { 2D EM simulation } \\
\text { efficiency }(\%)\end{array}$ \\
\hline-2 & 69.1 & 68.9 \\
-1 & 77.9 & 76.9 \\
0 & 87.2 & 87.4 \\
1 & 90.8 & 90.5 \\
2 & 88.4 & 86.7 \\
\hline \hline
\end{tabular}

design II are $Z_{\text {out }} / Z_{\text {in }}=16.05$ and $L=2063 \mathrm{~cm}$, which corresponds to $\tau=615 \mathrm{~ns}$ for a water-filled line or $\Gamma=$ 0.243. The sampled gap profiles are shown in Fig. 6 .

Results from calculations for transformer power efficiency as a function of input pulse length are shown in Fig. 7 for the exponential profile, linear profile, and $m=2$ profile from Fig. 6. Each curve approaches $100 \%$ efficiency in the short-pulse limit (as $\Gamma \rightarrow 0$ ) and each approaches the low-frequency result $(\Gamma \rightarrow \infty)$,

$$
\frac{P_{\text {out }}}{P_{\text {in }}}\left(\omega_{0} \rightarrow 0\right)=\frac{4 Z_{\text {in }} Z_{\text {out }}}{\left(Z_{\text {in }}+Z_{\text {out }}\right)^{2}}
$$

in the long pulse limit.

At intermediate values, $0<\Gamma<2$, the exponential profile has the highest efficiency of the three profiles considered. At values of $\Gamma>2$, the $m=0$ curve (linear profile) efficiency exceeds that of the exponential curve. It is shown below where intermediate values of $m$ are resolved that, for pulses with $\Gamma>0.5$, the optimum profile deviates significantly from an exponential.

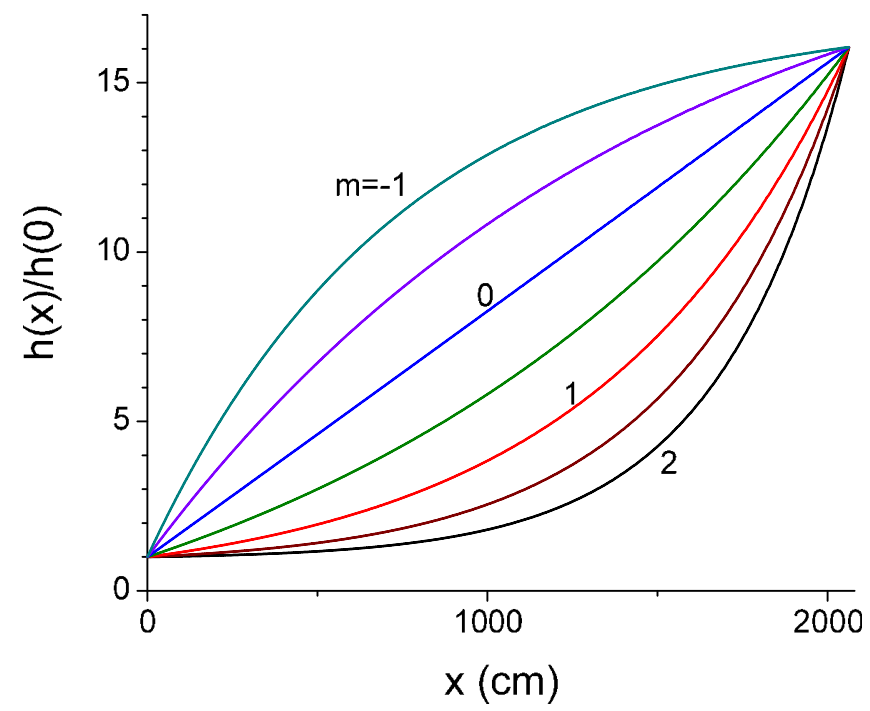

FIG. 6. (Color) Normalized planar-transmission-line gap profiles for design II for several values of the profile parameter $m$ (in steps of $\frac{1}{2}$ ).

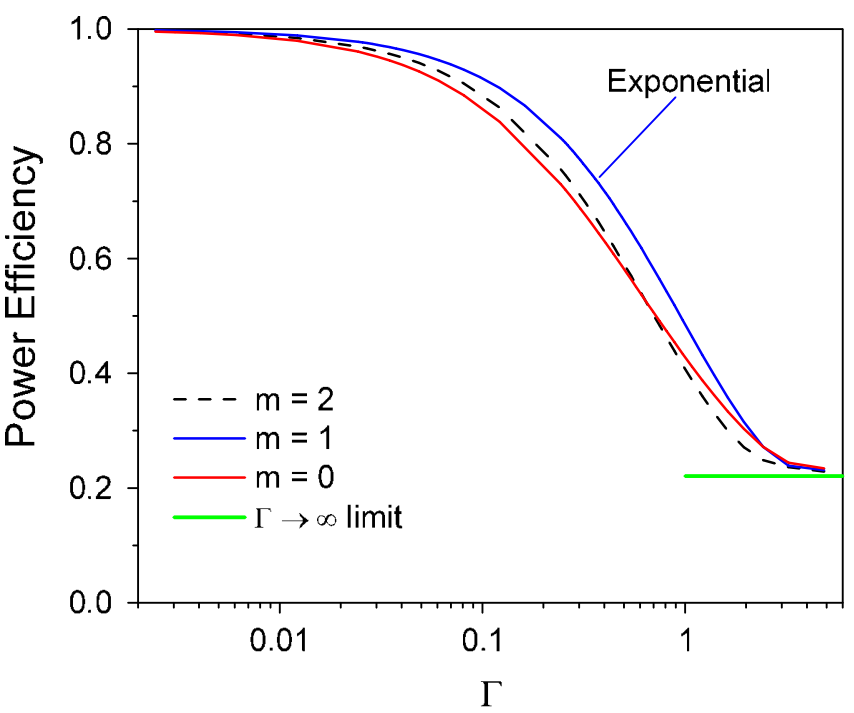

FIG. 7. (Color) The power transmission efficiency as a function of normalized input pulse width $\Gamma$ for the exponential $(m=1)$, linear $(m=0)$, and $m=2$ profiles.

As pointed out previously (e.g., Ref. [11]), the impedance transformation results in a shortening of the output voltage pulse in addition to a drop in peak power. This effect is illustrated in Fig. 8 for the exponential profile. The reduction of the voltage pulse width is a maximum of $\sim 15 \%$ which occurs near $\Gamma=1$; however, this compression comes at the expense of a $50 \%$ power transmission efficiency. Because the power efficiency decreases in time, the transported energy efficiency integrated to the time of peak power is always higher than the power efficiency (particularly important for $z$-pinch loads). Figure 8 is qualitatively consistent with Fig. 5 of Ref. [11] which

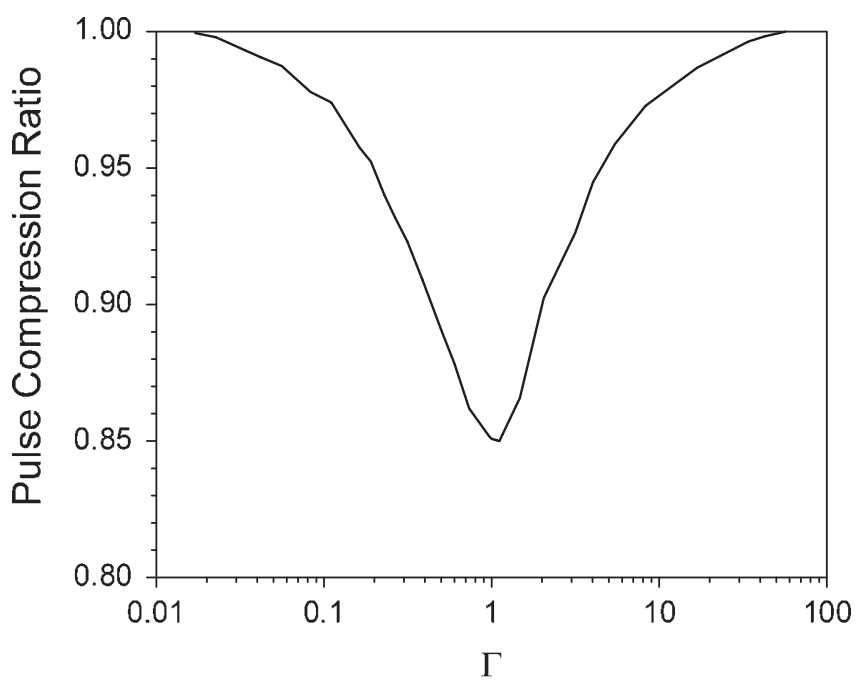

FIG. 8. The ratio of the output to input voltage pulse width for the exponential impedance profile as a function of the normalized pulse width $\Gamma$. 


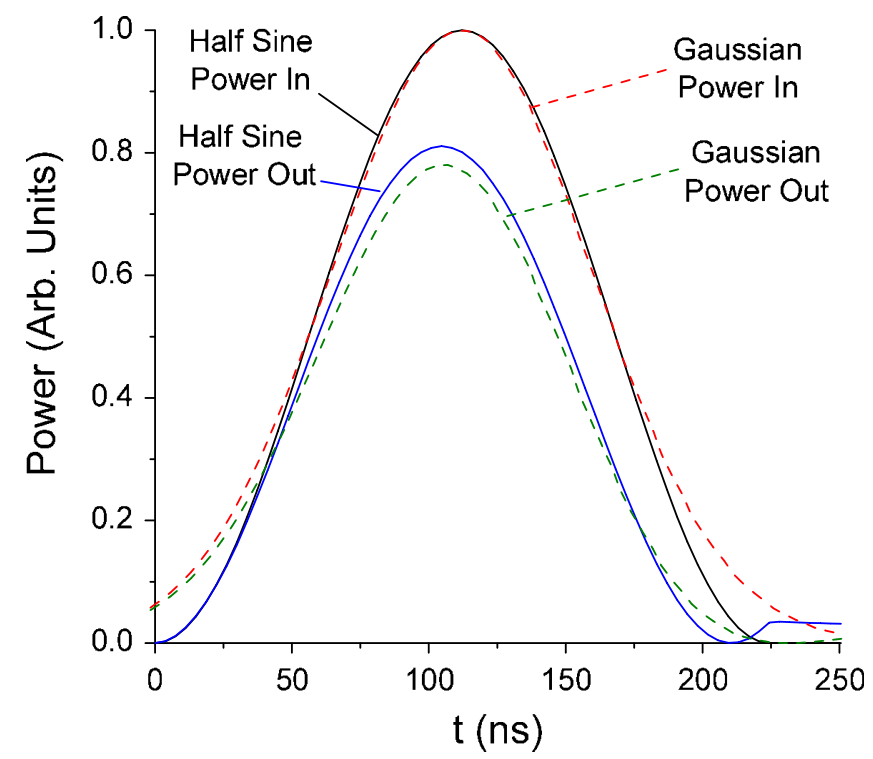

FIG. 9. (Color) The input and output power pulses for the halfsine and Gaussian shapes are compared for design II parameters with an exponential impedance profile.

shows results corresponding to Gaussian pulses of varying widths.

Input and output waveforms of both the half-sine and Gaussian pulses are compared in Fig. 9. The input Gaussian pulse width is chosen such that the FWHM of the input power pulse is the same as that of the half sine. The key difference between the two pulses is that the Gaussian has significant temporal wings. Because of reflections from the early portion of the pulse, the transported power for the Gaussian pulse deviates from that of the input at a lower power level. Thus, the Gaussian pulse

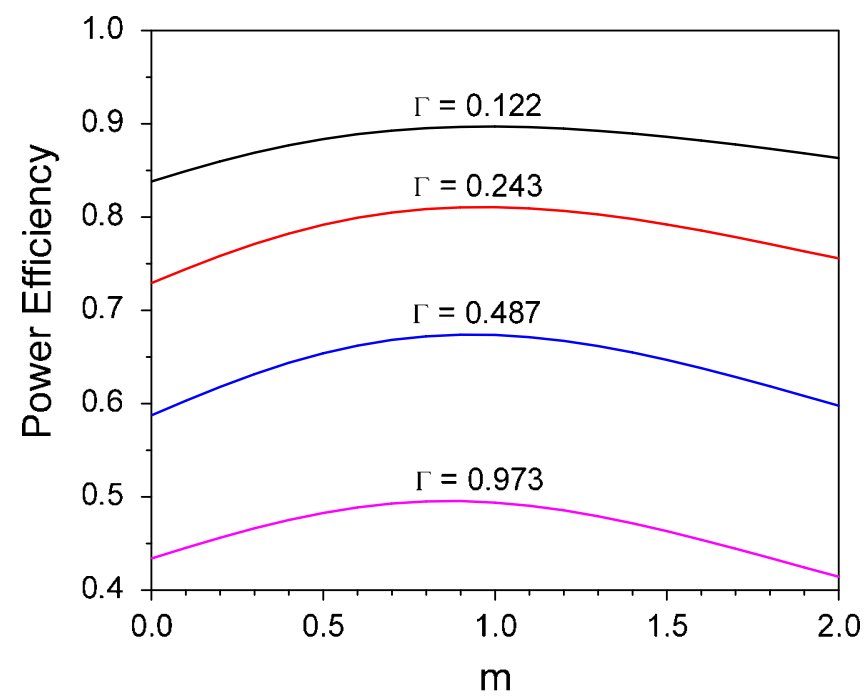

FIG. 10. (Color) The transmitted power efficiency as a function of profile parameter $m$ for several values of the normalized voltage pulse width $\Gamma$ at the input. has a 3\% lower power efficiency, but otherwise similar behavior, as the half-sine pulse.

In Fig. 10, we plot the power efficiency as a function of profile parameter $m$ for four values of $\Gamma$ for the half-sine pulse shape. For the shorter pulse width, the curves peak at

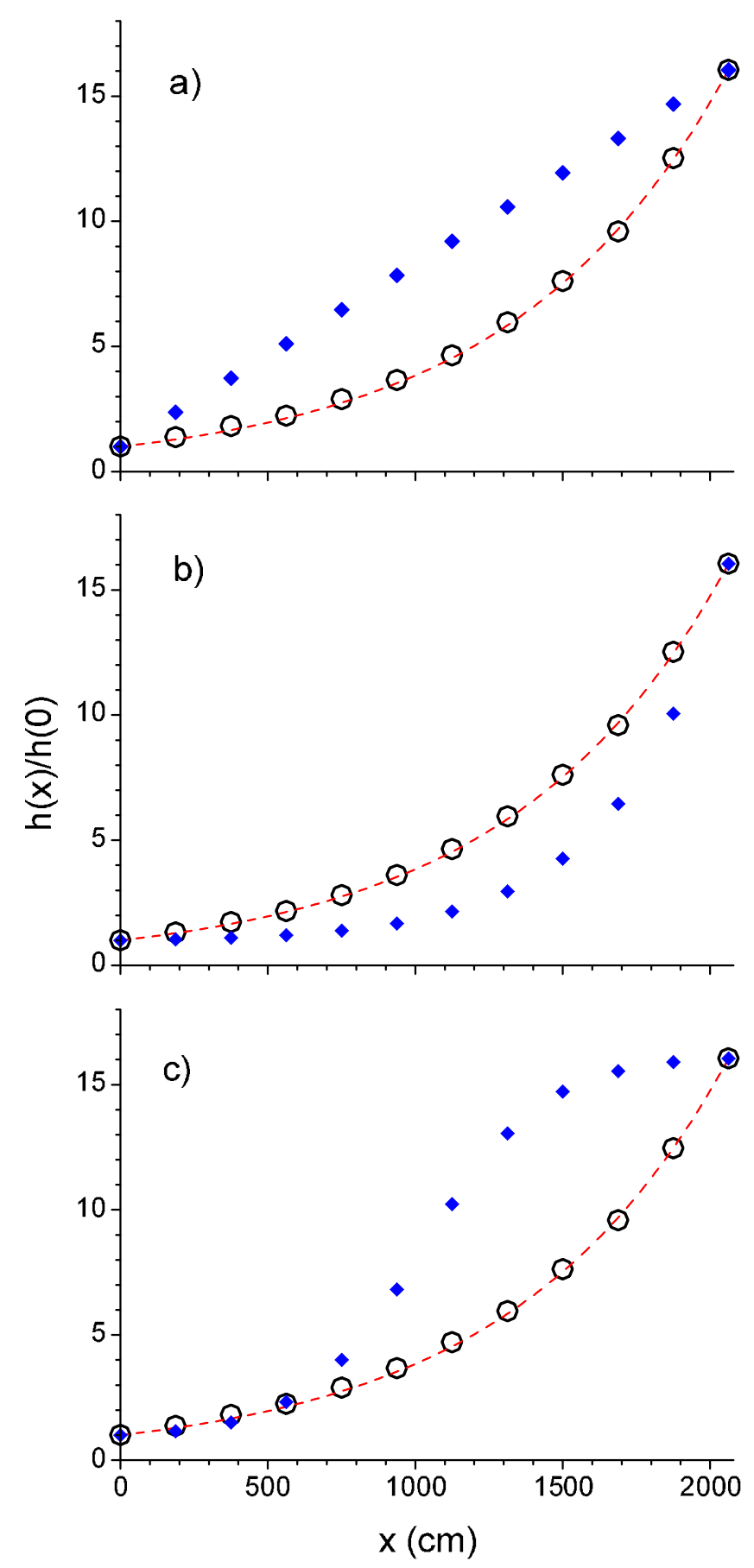

FIG. 11. (Color) Initial guess for gap optimization procedure $(\diamond)$, final optimized values $(\bigcirc)$, and the exponential curve (dashed line) are plotted. In (a), the initial guess is a $m=0$ (linear) profile; in (b), the initial guess is the $m=2$ profile; and in (c), the initial guess the profile from Eq. (15). 
or very close to $m=1$. For $\Gamma=0.487$, however, optimum power efficiency shifts to the left to $m \approx 0.9$, and continues to shift to values of smaller $m$ for even longer pulses. The corresponding differences in peak power efficiency are, however, small $(<1 \%)$ in this range of pulse width.

As a final demonstration of the optimal nature of the exponential profile, an optimization procedure was devised that allows for continuous variations in the impedance profile for a number of discrete elements. For these calculations, the profile is represented approximately by fixing the end point impedances $\left(Z_{\text {in }}\right.$ and $\left.Z_{\text {out }}\right)$ and allowing for 10 variable impedance values at uniformly spaced $x$ positions. The total of 12 functional values formed the basis for a look-up tabular function which uses quadratic interpolation for the intermediate values of $x$ on the 12 node grid. An input voltage pulse with $\omega_{0}=1.4 \times 10^{7} \mathrm{~s}^{-1}(\Gamma=0.243)$ from Eq. (5) is used. From an arbitrary initial guess for the 10 functional values, a multivariable (Powell's method) optimization procedure [13] is used to iterate toward a solution corresponding to maximum transmitted power.

Figures 11(a)-11(c) shows the results for three different initial guesses. In Figs. 11(a) and 11(b), the initial guesses corresponded to the $m=0$ (linear) and $m=2$ profiles, respectively. In Fig. 11(c), the initial guess is based on a profile that connects smoothly in slope to the constantimpedance inlet and outlet matching sections, i.e.,

$$
Z(x)=Z_{\text {in }}+\frac{Z_{\text {in }}+Z_{\text {out }}}{2}\left[1+\frac{\tanh \left(\alpha\left(\frac{x}{L}-\frac{1}{2}\right)\right)}{\tanh \frac{\alpha}{2}}\right]
$$

with $\alpha=5$.

As is clear from Fig. 11, in each case the procedure converges to a set of values very close to the exponential profile, and is optimized to within $0.1 \%$ of the exponential transmitted power efficiency. The optimization calculations were repeated for a shorter pulse width $(\Gamma=$ 0.122 ), and again convergence to a near-exponential profile is obtained. For reasons stated earlier, it is impossible to generalize to all pulse shapes and transformer designs, but certainly for the designs considered here and $\Gamma \leq 0.5$, the exponential impedance profile is for all practical purposes optimal. Nevertheless, it is clear that the approach outlined in this section can be used to determine the optimum impedance profile of a transformer, for any given pulse shape and width.

\section{SUMMARY AND CONCLUSIONS}

We find in semianalytic theory and 2D simulation that an impedance transformer can provide reasonable voltage transformation and high transmission efficiency for pulsed-power applications. We also present the first 2D simulations that verify the quasi-TEM approximation of a semianalytic transmission-line model, giving agreement to within $1 \%$ over a wide range of impedance profiles.

In the short-pulse limit, the power transmission efficiency approaches unity for all transformers, generally a huge improvement over that of a sudden change $(\Gamma \gg 0)$ in impedance from initial to final impedances. Using an optimization procedure, we find that in the short-pulse limit, an exponential impedance profile offers the most efficient transmission but slightly deviates from optimal for longer pulses, i.e., $\Gamma>0.5$. The exponential profile results in a constant $\Delta Z / Z$ along its entire length leading to the smallest level of wave reflections per unit length. We have also developed a procedure that can be used to determine the optimum impedance profile, for an arbitrary pulse shape and width.

An impedance transformer can also be used to sharpen the incoming power pulse. We find, however, that this characteristic is optimized for $T / \tau$ near unity where the power transmission efficiency is likely too low to be of interest. Nevertheless, a transformer would reduce significantly the amplitude of a low-frequency prepulse as suggested in Ref. [1].

The modeling has been applied to two published pulsedpower designs for a petawatt-class $z$-pinch driver. We find the results are in good agreement with those of Ref. [1] with transformer efficiencies as high as $91 \%$. The results presented here are for ideal 2D geometries. In future work, we will examine the practical application of the transformer in 3D in a radial line as well as the associated nonideal coupling at the ends of the transmission line.

\section{ACKNOWLEDGMENTS}

The authors would like to thank Sue Welch for her assiduous technical editing of this manuscript. This work was supported by Sandia National Laboratories. Sandia is a multiprogram laboratory operated by Sandia Corporation, a Lockheed Martin Company, for the U.S. Department of Energy's National Nuclear Security Administration under Contract No. DE-AC04-94-AL85000.

\section{APPENDIX: NUMERICAL TRANSMISSION-LINE REPRESENTATION OF AN IMPEDANCE TRANSFORMER}

Commonly, transmission-line codes such as BERTHA [16] are used to aid the design of large-scale pulsed-power generators. We briefly compare the results of a simple transmission-line representation of an exponential impedance transformer with the more rigorous model presented in Sec. II. For simplicity, we choose to represent the impedance transformer as a finite number of fixed length transmission-line elements. For an impedance transformer with an exponential profile, the ratio of the change in impedance to the local impedance value is a constant, $\beta$. Therefore, the impedances for the individual elements are given by the iterative equation,

$$
Z_{i+1}=(1+\beta) Z_{i}
$$

where $Z_{i}$ is the impedance of the $i$ th transmission-line 


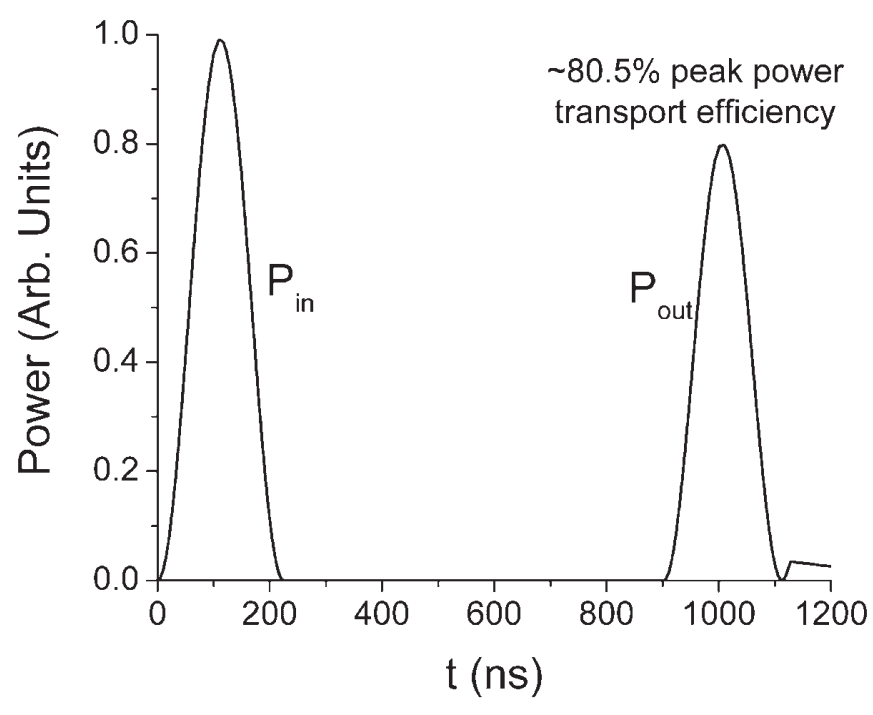

FIG. 12. Incident $\left(P_{\text {in }}\right)$ and transmitted $\left(P_{\text {out }}\right)$ power pulse waveforms from an exponential impedance transformer (design II) as represented by 41 elements in the transmission-line model.

element. For an $n$-element series with $Z_{1}=Z_{\text {in }}$ and $Z_{n}=$ $Z_{\text {out }}$ (both known values), expression (A1) represents a series of $n-1$ coupled equations in $n-1$ unknowns $\left(Z_{2}, \ldots, Z_{n-1}, \beta\right)$ that can be solved to find $\beta$ and therefore all of the element impedances. The value of $\beta$ is simply

$$
\beta=\left(\frac{Z_{\text {out }}}{Z_{\text {in }}}\right)^{1 /(n-1)}-1 .
$$

For direct comparison with the results presented in Sec. II, we carried out a sample impedance-transformer calculation using a simple transmission-line code contained within the LSP code. The impedance-transformer section parameters are identical to the example used in Sec. IV design II: $Z_{\text {in }}=0.0162 \mathrm{Ohms}, Z_{\text {out }}=0.26 \mathrm{Ohms}$, $L=2050 \mathrm{~cm}$, and $\varepsilon=80$. The transmission-line representation of the impedance transformer used 41 elements ( $\beta \sim 0.071856$ ), each $50-\mathrm{cm}$ in length, driven by a onehalf period sinusoidal voltage pulse $(T / \tau=0.243$ or $\sim 225 \mathrm{~ns}$, zero-to-zero width). The transmission-line model also included very long, constant-impedance sections at the inlet and outlet to isolate the incident and transmitted power pulse, and track any numerical signal dispersion. The incident and transmitted power through the impedance transformer is shown in Fig. 12. The transmitted peak power efficiency obtained for this 41-element representation is $\sim 80.5 \%$, in very good agreement with the model of Sec. II (see Fig. 8, for curve $T / \tau=0.243$ at $m=$
1). Both results also agree, to within $<1 \%$, with those presented in the fourth row of Table III of Ref. [1].

[1] W. A. Stygar, M. E. Cuneo, D. I. Headley, H. C. Ives, R. J. Leeper, M. G. Mazarakis, C. L. Olson, J. L. Porter, T. C. Wagoner, and J.R. Woodworth, Phys. Rev. ST Accel. Beams 10, 030401 (2007).

[2] A. A. Kim, A. NB. Bastikov, S. N. Volkov, V. G. Durakov, B. M. Kovalchik, and V. A. Sinebryukhov, in Proceedings of the 14th International Conference on High-Power Particle Beams (Beams 2002), edited by T. A. Mehlhorn and M. A. Sweeney (AIP, Melville, New York, 2002), pp. 81-84.

[3] A. A. Kim, A. NB. Bastikov, S. N. Volkov, V. G. Durakov, B. M. Kovalchik, and V. A. Sinebryukhov, in Proceedings of the 14th IEEE International Pulsed Power Conference Technical Digest, edited by M. Giesselman and A. Neuber (IEEE, Piscataway, NJ, 2003), pp. 853-854.

[4] C.L. Olson, in Landholdt-Boernstein Handbook on Energy Technologies, edited by W. Martienssen, Fusion Technologies Vol. VIII/3, edited by K. Hienloth (SpringerVerlag, Berlin-Heidelberg, 2005).

[5] J. A. Maenchen, G. Cooperstein, J. O'Malley, and I. Smith, Proc. IEEE, 92, 1021 (2004).

[6] J. D. Sethian et al., Proc. IEEE 92, 1043 (2004).

[7] R. Spielman et al., in Proceedings of the 13th IEEE International Pulsed Power Conference, edited by G. Cooperstein and I. Vitkovitski (IEEE, Piscataway, NJ, 1997), p. 698.

[8] P. W. Smith, Transient Electronics (John Wiley \& Sons, West Sussex, England, 2002) Chap. 6, and references therein.

[9] I. A. D. Lewis and F.H. Wells, Millimicrosecond Pulse Techniques (Pergamon Press, New York, 1959).

[10] K. Pendergraft and R. Pieper, J. Acoust. Soc. Am. 94, 580 (1993).

[11] Y.P. Tang and Z. Li, IEEE Trans. Microwave Theory Tech. 43, 2573 (1995).

[12] C. G. Montgomery, R.H. Dicke, and E. M. Purcell, Principles of Microwave Circuits (McGraw-Hill, New York, 1948).

[13] W. H. Press, B.P. Flannery, S. A. Teukolsky, and W.T. Vetterling, Numerical Recipes (Cambridge University Press, New York, 1989).

[14] T. P. Hughes, S.S. Yu, and R.E. Clark, Phys. Rev. ST Accel. Beams 2, 110401 (1999).

[15] D. R. Welch, D. V. Rose, R. E. Clark, T. C. Genoni, and T. P. Hughes, Comput. Phys. Commun. 164, 183 (2004).

[16] D. D. Hinshelwood, Naval Research Laboratory Memorandum Report No. 5185, 1983. 